\title{
Image Restoration Methods, Survey of Machine Learning Methods and an Over View
}

\author{
G.S. Yogananda \\ Department of Electronics and Communication \\ Kalpataru Institute of Technology, Tiptur \\ Karnataka, India-572201, VTU, Belagavi-590018
}

\author{
Y.P. Gowramma \\ Department of Computer Science and Engineering \\ Kalpataru Institute of Technology, Tiptur \\ Karnataka, India-572201, VTU, Belagavi-590018
}

\begin{abstract}
Image restoration is one of the crucial problems in image processing even it is the low level image processing activity. This research paper presents the overview of the image restoration, available standard noises, and sources of noises. Further it details the degradation model, available restoration techniques, medical image restoration and medical restoration techniques. The literature survey on machine learning in restoration is explored. Finally mentioned the research gap to carry out the further research.
\end{abstract}

\section{Keywords}

Restoration, Noise, Machine Learning

\section{INTRODUCTION}

A real world scenario works more with the image and video than other data's. Acquisition of images and video's involves numerous noises. Majorly defocus and the motion blur. This involves the image processing activity. Image restoration is the one of the central problem in the low level image processing. The restoration process removes the noise and reduce the degradation in the images using various methods comes under Image Restoration. Restoration is the one of the important process for next level activities such as segmentation, feature extraction and other higher level activities. Noise free images leads to the higher accuracy in the higher level activities. This paper concentrates on the machine learning algorithm based restoration. Degradations may be due to sensor noise, blur due to camera misfocus, relative motion between camera and object, atmospheric turbulence, and other reasons. Using the prior knowledge of degradation phenomena, restoration can be applied to a degraded image to restore or reconstruct the image. Here degradation is modeled as a linear, position invariant process followed by additive noise that is not correlated with image values and the inverse process is applied in order to recover the original image. The applications of restoration are in photography, forensic images, satellite images, motion sequence images, majorly in medical images, identification, computer vision or robot vision, steganography, image enhancement, image analysis in medical images, morphological image analysis, space image analysis, bottling and in industry.

\section{DEGRADATION AND RESTORATION MODEL}

The Image processing technique we enhance the corrupted input image data (raw images) captured from the cameras is placed on the different fields of the life for various mentioned applications. The results of the restored images are of the greater quality as well as the objects which are clearly visible as comparable to the initially sensed images. There are various primary steps involved in the image processing that are representation of images, preprocessing of images, enhancement, restoration, analysis, reconstruction of images and image data compression.

Image Restoration: The image restoration concerns about the noise removal or reduction which are included during the acquisition of images and many other stages. e.g. Noise, pixel value errors, misfocus blurring or camera motion blurring using prior knowledge of the degradation phenomenon. This means it deals with the modeling of the degradation and applying the process (Inverse) to reconstruct the image. The image noise removal has got a several applications in the fields such as image processing, pattern recognition, machine learning, computer vision and robotics.

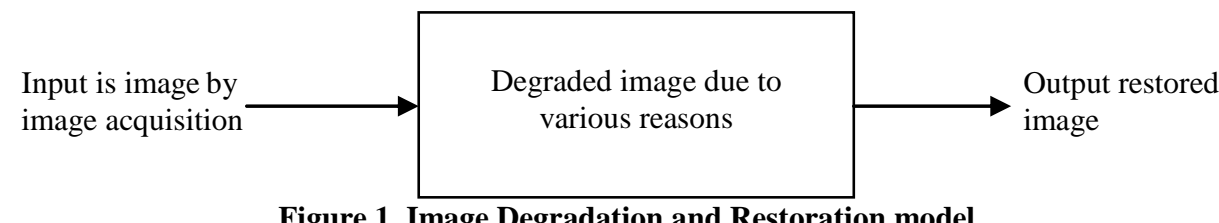

Figure 1. Image Degradation and Restoration model

Figure 1 shows the image degradation and restoration model. Here input is image captured by imaging system. Captured images undergo various noises. Output is the restored image.

\section{SOURCES OF NOISES}

Noise in digital images is during image acquisition and/or Transmission. The sensors performance will be affected by variety of factors like environmental conditions during image acquisition and by the quality of the sensing elements themselves. During transmission, images are corrupted by the noises due to interference in the channel used for the transmission. Prior knowledge of noise models is required for eliminating them. A better restoration can be made if the noise model is known.

Some important Noise PDFs are

Gaussian Noise - Arises in an image due to the electronic noise and sensor noise due to poor illumination and/or high temperature.

Raleigh Noise - is helpful in characterizing noise phenomena 
in range imaging.

Erlang (Gamma) Noise - finds application in laser Imaging

Exponential Noise - finds application in laser Imaging.

Uniform Noise - least descriptive.

Impulse (Salt \& Pepper Noise) Noise - is found in situations where quick transients, such as faulty switching take place during imaging.

Periodic noise - this is a special kind of noise which has to be eliminated in frequency domain.

The above Noise PDFs can be used to Model the noise corruptions found in the image.

\section{Sources of Blur}

Uniform linear motion between the image and the sensor during acquisition, camera shutter operation, blur due to misfocus,

\section{TRADITIONAL IMAGE RESTORATION TECHNIQUES}

Image restoration Techniques can be classified into Deterministic \& Stochastic methods. Deterministic methods are subdivided into Linear \& Nonlinear methods. Deterministic methods can be applied if prior knowledge of degradation is known. Stochastic methods are those if prior knowledge of degradation is not known. Linear methods are those where PSF of the degradation system is known. But here effect of noise is not considered. PSF(point spread function) is the degree to which an optical system blurs (spreads) a point of light.

Image restoration techniques can also be categorized into Blind Deconvolution \& Non-Blind deconvolution methods depending on the knowledge of the PSF. Blind methods are those when a little or no knowledge of PSF is known whereas Non-blind deconvolution is when the knowledge of PSF is known.

For image restoration when only additive noise is present can be performed using many spatial filters like Mean filters under which we have Arithmetic filters in which the noise is reduced as a result of blurring, Geometric mean filters using which smoothing is achieved comparable to arithmetic filters, but some image details are lost, Harmonic filters which works well for salt noise \& also for Gaussian noise, Contra harmonic mean filter which is virtually used for eliminating the effects of salt \& pepper noise. Order statistics filters are also used under which we have Median filters which are used for eliminating random noise with considerably less blurring as compared to linear filters of the same size, Max and Min filters to reduce pepper \& salt noise respectively, Midpoint filter which works better for randomly distributed noises like Gaussian noise or Uniform noise, Alpha Trimmed mean filter to remove salt-and-pepper noise and Gaussian noise, Adaptive filters whose behavior changes based on statistical characteristics of the image within the window.

Degradation is modeled and deconvolution filters are used for image restoration. Degradation function can be estimated by one or the other way and the process of restoring an image by using the estimated degradation function is called a Blind Deconvolution. Inverse filtering can also be used for restoration of images degraded by degradation function. In inverse filtering no explicit provision for handling noise is available. Wiener (Minimum mean square error filter) filtering can be used in which both degradation function \& the statistical characteristics of the noise are incorporated into the restoration process. But here the power spectra of the non degraded image and the noise must be known. Wiener filtering is a Non-blind procedure. Constrained Least Squares filtering can be used which requires only the mean $\&$ the variance of the noise is required.

\section{MEDICAL IMAGE RESTORATION}

There are a number of medical images which are $\mathbf{X}$-ray images, Ultrasound images, CT scan Images, MRI images etc.

\subsection{Medical Image computing}

Medical imaging is increasingly being used as a first step for clinical diagnosis of a large number of diseases, including disorders of the brain, lung, kidney, muscle etc. Many imaging techniques are present for analyzing the abnormalities if present. However subtle changes in the images are difficult to locate for the human eye. Hence advanced image processing techniques can be of great medical value to assist the radiologist as well as to understand the basic mechanism of action in a large number of diseases. Further early diagnosis is critical for better clinical outcome.

\subsection{Noises in Medical Images}

The most commonly affected noises in medical MRI image are Salt and Pepper, Speckle, Gaussian and Poisson noise. In the field of medical imaging \& applications many traditional noise removal techniques/ algorithms like wiener filter to filter the noise present in an image which consists corrupted signal in it, Gaussian filter to remove Spackle noise in ultrasound Images or MRI images, median filter to remove impulse noise etc are adopted. It has been found that the median filter performs better for removing salt-and-pepper noise and Poisson Noise for images in gray scale, and Weiner filter performs better for removing Speckle and Gaussian Noise and Gaussian filter for the Blurred Noise.

\section{MACHINE LEARNING METHODS FOR IMAGE PROCESSING}

Machine learning is a branch of AI, a component of human intelligence, which is an interesting field of research. Machine learning can be used in medical images for prediction or medical diagnosis. With the help of machine learning the computers can perform the tasks tirelessly \& consistently. Machine learning as applied to Image processing mainly consists of algorithms for computing image features which are important in making the prediction or diagnosis of interest.

Some of the machine learning algorithms is neural networks, k-nearest neighbors, support vector machines, decision trees, the naïve Bayes algorithm and deep learning. Machine learning is already being applied in the practice of Radiology and these applications will probably grow at a rapid pace in the near future. Many open source tools for developing and implementing machine learning algorithms which are compatible with majority of modern programming languages including like Python, C++, MATLAB, R and Lua.

\section{LITERATURE SURVEY ON MACHINE LEARNING AS APPLIED TO IMAGE PROCESSING}

Giacomo Boracchi and Alessandro Foi[1,2012] are detailed a methodology for deriving a statistical model of the performance of a given deblurring alogorothm, when used to 
resotre motion blurred images. They deal with random motion, which is effectively handled by means of statistical descriptors of the PSF.

[2] Prabhishek Singh \& Raj Shree[2016] explored about maximum noise models are covered that gives a detailed understanding of all the perspectives of the noise including their advantages and disadvantages . Various sources of noises and applications of image restoration are discussed. Apart from noise a detailed comparative study of image restoration and filtering techniques is also given that helps the new researchers to understand the various aspects of the image restoration that will create interest and lead them to work in specified hybrid technique.

[3] Anamika Maurya \& Rajinder Tiwari[2014] expressed the objectives and definition of image restoration is addressed . They also give a brief introduction about various blurring and deblurring techniques. Each technique has its own advantages and disadvantages. This work will be helpful for 'the researchers in understanding the concept of image restoration and deblurring techniques who are new in this field. Research work is taking place on image fusion techniques using two algorithms, but there is scope for the improvement. Here more than two algorithms and more samples can be used results could have been better.

[4] Shilpa Rani, Sonika Jindal, Bhavneet Kaur[2016] identified many deblurring techniques. After conducting literature survey on various image deblurring techniques proposed by different researchers. Restoration or deblurring blur from images is a different problem to resolve. It is concluded from above techniques that lucy and wiener filter give better results as compare to other techniques. The research gap is on further advancement try to apply neural network on these techniques. These results are concluded on the basis of MSE and PSNE parameters.

P.Sumitra[5,2016] studied a method for blind deconvolution algorithm. To conclude restoration of medical images like Xray, CT, MRI, and ultrasound is very crucial and sensitive matter. The restoration must be done to maximum possible level. The PSNR value of inverse filter is poor when compared with that of weiner filter. Reaserch gap is to develop and build improved technique which will give better performance than weiner. Explore using machine learning and pattern recognition technique is open problem of research.

Christian J. Schuler et a[9,2013] shown that neural networks achieve a new state of the art in image deconvolution. Here all scenarios are tested. This presents a clear benefit in that it is learning. It do not need to design or select features or even decide on a useful transform domain, the neural network automatically takes care of these tasks. The limitation of this approach is that each MLP has to be trained on only one blur kernel. Results achieved with MLPS trained on several blur kernels are inferior to those achieved with MLPs trained on a single blur kernel. This makes this approach less useful for motion blur, which are different for every images. However, in this case the deblurring quality is currently more limited by errors in the blur estimation than in the non-blind deconvolution step possibly this method could be further improved with a meta-procedure.

Ying Shen and Weihua Zhu[11,2013]presents an approach for medical color image processing(take the heart images obtained by special instrument for example).this approach has four steps. For the first processing the smoothing algorithm is to remove noise and artifacts in the image. Then smooth the images is addressed by the filter algorithm which performs in the filtering process. The use of the image distance difference method is to increase the filtering effect. Subsequently, the image enhancement algorithm uses weighted enhanced imagers of different regions. Finally, restoration algorithm is used to restore the color images of the heart. The experimental results show that the proposed four-step method can be effectively used to achieve a good image expected effect.

The future research work (research gap) directions may be carried out from several aspects. First, the experimental use of the sample images will do the comparison algorithm for multi-capacity effect pending further study. Second, the image restoration algorithm for medical image information of the entire heart is effectively. But changes in the heart muscle gradient needs detailed study. Finally, connection analysis about heart, and other organs, such as move, is inadequate in this research. Therefore, the aspects of the proposed algorithm and the way to in depth reaerch or extension applications could be studied in the future.

[13] Komal Sharma et al[2014] proposes a work on brain tumor detection system based on machine learning algorithms. The texture based features are extracted using Gray level cooccurrence matrix(GLCM). The texture feature features of the image considered in this proposed work includes energy, contrast, correlation, homogeneity and Naïve bayes machine learning algorithms is used and the maximum accuracy $98.6 \%$ and $91.0 \%$ is achieved by considering 212 samples of brain MR images. The feature work is the accuracy can probably be increased by considering a large data set and extracting intensity based features in addition to the texture based features.

Bradley J. Erickson et al[14,2017] explored machine learning is already being applied in the practice of radiology, and at a rapid pace in the near future. The use of machine learning in radiology has important implications for the practice of medicine, and it is important that they engage this area of research in ensure that the best care is afforded to patients. Understanding the properties of machine learning tools is critical to ensuring that they are applied in the safest and most effective manner.

Nalin kumar et al[15,2017] work taken twenty different medical images like MRI for doing the experiment for noise removal. They added salt pepper, Gaussian, speckle, blurred and poison noise to the images and also removed these noises from the above medical images by

\section{Research gap or open problem in image restoration}

The survey reveals the many new direction to the research. The researchers considered the traditional methods of the restoration. The artificial intelligence and the Machine learning involve many computation techniques. Survey explores the deep learning in restoration. Since other different kinds of neural networks and fuzzy techniques, unsupervised techniques and probabilistic methods in restoration are still open problems in phographic restoration, medical image restoration, biometric restoration, forensic restoration and scanned document restorations. . As the imaging technology advances the new problems of restoration may arises as the open research problems.

\section{CONCLUSION}

This research paper presents the overview of the image restoration, available noises, and source of noises, available restoration techniques, and restoration techniques, the literature survey and finally explore about the research gap 
and open area of the research.

\section{REFERENCES}

[1] Giacomo Boracchi and Alessandro Foi , "Modeling the Performance of Image Restoration from Motion Blur" IEEE Transactions on Image Processing, Volume: 21 , Issue: 8 , Aug. 2012 , Page(s): 3502 - 3517.

[2] Prabhishek Singh \& Raj Shree, "A Comparative Study to Noise Models and Image Restoration Techniques", International Journal of Computer Applications (0975 8887) Volume 149 - No.1, September 2016.

[3] Anamika Maurya \& Rajinder Tiwari, "A Novel Method of Image Restoration by using Different Types of Filtering Techniques", International Journal of Engineering Science and Innovative Technology (IJESIT) Volume 3, Issue 4, July 2014.

[4] Shilpa Rani, Sonika Jindal, Bhavneet Kaur, "A Brief Review on Image Restoration Techniques", International Journal of Computer Applications, Foundation of Computer Science (FCS), NY, USA, Volume 150 - Number 12, Year of Publication: 2016.

[5] P.Sumitra, "A Comparative study algorithm for Noisy Image Restoration in the field of Medical Imaging", International Journal of Advanced Information Technology (IJAIT) Vol. 6, No. 1, February 2016.

[6] Ishfaq Bashir et al, "Image Restoration and the various Restoration Techniques used in the field of Digital Image 'Processing", International Journal of Computer Science and Mobile Computing, Vol.6 Issue.6, June- 2017, pg. 390-393.

[7] Mohd. Junedul Haque \& Churu, "A Brief Review of Image Restoration Techniques", International Journal of Advanced Computing Research ISSN [2349-7130],
Volume 01, 2014.

[8] Monika Maru, M. C. Parikh, "Image Restoration Techniques: A Survey", International Journal of Computer Applications Foundation of Computer Science (FCS), NY, USA, Volume 160 - Number 6 Year of Publication: 2017.

[9] Christian J. Schuler et al, "A Machine Learning Approach for Non-blind Image Deconvolution”, 2013 IEEE Conference on Computer Vision and Pattern Recognition, 23-28 June 2013.

[10] Liyang Wei ; Yongyi Yang; R.M. Nishikawa; Yulei Jiang, "A study on several Machine-learning methods for classification of Malignant and benign clustered microcalcifications", IEEE Transactions on Medical Imaging, Volume: 24 , Issue: 3 , March 2005.

[11] Ying Shen and Weihua Zhu, "Medical Image Processing using A Machine Vision-based Approach", International Journal of Signal Processing, Image Processing and Pattern Recognition Vol. 6, No. 3, June, 2013.

[12] Ruohan Gao \& Kristen Grauman, "On-Demand Learning for Deep Image Restoration", Published in IEEE International Conference on Computer Vision...2017

[13] Komal Sharma et al, "Brain Tumor Detection based on Machine Learning Algorithms", International Journal of Computer Applications (0975 - 8887) Volume 103 No.1, October 2014.

[14] Bradley J. Erickson et al, "Machine Learning for Medical Imaging”, Radiographics, vol 27, No 2, feb 172017.

[15] Nalin kumar et al, "Noise Removal and Filtering Techniques used in Medical Images", Oriental Journal of Computer Science \& Technology, March 2017, Vol. 10, No. (1), Pgs. 103-113. 